\title{
因HAD
}

ISSN-L: 2530-5115

DOI: http://doi.org/10.22585/hospdomic.v3i1.59

\section{Manejo de la insuficiencia cardíaca refractaria a diuréticos con suero salino hipertónico}

\author{
Management of diuretic refractory heart failure with \\ hypertonic saline solution
}

Cristina Soriano Maldonado', Rafael López-Bas Valero', Annabella Freitas Amorós

1. Unidad de Hospitalización a Domicilio. Hospital San Juan de Alicante. España

Correspondencia/Correspondence

Cristina Soriano Maldonado

Unidad de Hospitalización a Domicilio.

Hospital San Juan de Alicante. España

cristina.sori.maldo@gmail.com

Recibido/Received

10.11.2018

Aceptado/Accepted

29.12 .2018
Conflicto de Intereses/Competing interest

Los autores declaran la inexistencia de conflicto de interés

CÓMO CITAR ESTE TRABAJO | HOW TO CITE THIS PAPER

Soriano Maldonado C, López-Bas Valero R, Freitas Amorós A. Manejo de la insuficiencia cardiaca refractaria a diuréticos con suero salino hipertónico. Hosp Domic. 2019;3(1):51-6. 


\section{RESUMEN}

La insuficiencia cardiaca es una de las patologías más prevalentes en nuestra sociedad, siendo una causa frecuente de morbimortalidad en ancianos. El tratamiento de la insuficiencia cardiaca aguda se basa principalmente en la administración de diuréticos de asa de forma intravenosa, sin embargo, en algunos casos su uso no se asocia a una mejora en la sintomatología ni el pronóstico, requiriendo reingresos hospitalarios de forma habitual. Presentamos el caso clínico de un paciente ingresado a cargo de la Unidad de Hospitalización a Domicilio que presentaba insuficiencia cardiaca crónica y edemas refractarios en ambos miembros inferiores a pesar de un tratamiento diurético adecuado. Se inicia infusión de suero salino hipertónico junto con altas dosis de furosemida, registrando una mejora de la sintomatología y reducción de los edemas manteniendo su función renal.

Palabras clave: Insuficiencia cardiaca; Edema; Salino Hipertónico; Furosemida.

\section{ABSTRACT}

Heart failure is one of the most prevalent diseases in our society, being a frequent cause of morbidity and mortality in elderly people. Treatment of acute heart failure is mainly based on the administration of intravenous loop diuretics; however, in some cases their use is not associated with an improvement of symptomatology or prognosis, frequently requiring readmission in a hospital. This is a case report about a patient who was admitted to San Juan de Alicante Home Hospitalization Unit. Despite a proper diuretic treatment, this patient had heart failure and refractory oedema in lower limbs. We initiate treatment with an infusion of hypertonic saline solution and high doses of furosemide, registering an improvement of the symptoms and reducing oedema maintaining renal function.

Keywords: Heart failure; Oedema; Hypertonic saline solution; Furosemide 


\section{INTRODUCCIÓN}

El aumento demográfico en las últimas décadas en los países desarrollados, está alcanzando tasas de crecimiento del 10\% en la población de más de 70 años. Este hecho supone en sí mismo un reto sanitario de primer orden, especialmente acuciado en el caso de pacientes con agudizaciones de su insuficiencia cardiaca (IC). Según el estudio PRICE, la prevalencia de la IC en nuestro país está en torno al 6,8\% de la población mayor de 45 años. Esta prevalencia es similar en varones y mujeres y aumenta de forma significativa con la edad, de tal forma que por encima de los 75 años se sitúa en el 16\%" La Sociedad Europea de Cardiología (European Society of Cardiology o ESC), prevé que la prevalencia de la IC se incrementará en un 25\% para el $2030^{2}$ debido al envejecimiento de la población y al aumento de los factores de riesgo (diabetes, hipertensión, obesidad,...) En España, la ICA es la primera causa de hospitalización en mayores de 65 años, representando cerca de 113.327 ingresos hospitalarios por año, de los cuales una cuarta parte mueren al año de su ingreso ${ }^{3}$. Este hecho supone un elevado uso de recursos y carga económica para la sociedad, dado que el $70 \%$ del coste económico de la ICA está relacionado con las hospitalizaciones. En España, la estancia media hospitalaria de un paciente que ingresa con ICA es de 7 a 10 días; y cada hospitalización cuesta al sistema sanitario 4.900€. Se estima que entre el 8-9\% del coste se podría ahorrar con una terapia que redujera la duración de la estancia hospitalaria a un día4.

Los datos europeos más recientes (estudio piloto ESC-HF) demuestran que las tasas de mortalidad por todas las causas a los 12 meses de los pacientes hospitalizados o estables ambulatorios con IC son del 17 y el 7\% respectivamente, y las tasas de hospitalización a los 12 meses fueron del 44 y el $32 \% 5$

Dada la mayor biodisponibilidad de los diuréticos cuando se administran por vía intravenosa (i.v.) en vez de por vía oral, el tratamiento hospitalario inicial de la ICA descompensada se sustenta fundamentalmente en la administración de diuréticos de asa de forma parenteral. Esto permite que el proceso de diuresis se inicie entre 30 y 60 min tras la administración (se produce una pérdida inicial en el sodio corporal total y posteriormente se desarrolla un mecanismo de adaptación fisiológico que conduce hacia un nuevo estado estacionario que trata de igualar la entrada y la excreción de sodio, lo que previene una excesiva perdida de sal y fluidos) Este enfoque tiene como objetivo mejorar la congestión pulmonar por el efecto dilatador venoso inicial, pero ha sido escasa la evaluación en ensayos clínicos aleatorizados de su eficacia para prolongar la supervivencia en la ICA. Así pues, el análisis del ensayo ESCAPE ${ }^{6}$ demostró que hay un riesgo de mortalidad dependiente de la dosis con los diuréticos i.v., especialmente con la furosemida si se superan los $300 \mathrm{mg} / \mathrm{día}$.

Aunque prácticamente el $90 \%$ de las ICA los reciben, la evidencia científica que apoya esta práctica es escasa actualmente y todavía existe cierta incertidumbre respecto a qué dosis y forma de administración es la óptima.

La resistencia a diuréticos, definida como la persistencia del edema a pesar de un tratamiento diurético adecuado (dosis de furosemida $\geq 160 \mathrm{mg} / \mathrm{d}$ de furosemida o equivalente, o $100 \mathrm{mg} / \mathrm{d}$ combinada con diuréticos tiacídicos y/o espironolactona) asociado a restricción del aporte hídrico y de sal, puede explicar porque hasta un $21 \%$ de los pacientes ingresados por ICA son dados de alta sin haber conseguido una pérdida de peso significativa o incluso con mayor peso que al ingreso ${ }^{7}$. La congestión refractaria es un reto en el tratamiento de la IC ya que la persistencia de datos congestivos se asocia a mayor mortalidad.

La resistencia a los diuréticos de asa y la preocupación por el empeoramiento de la función renal durante la agudización de la insuficiencia cardiaca pudiendo derivar en mayor mortalidad, ha llevado a buscar alternativas de tratamiento. En la última década se han desarrollado ensayos con la asociación de suero salino hipertónico y dosis elevadas de furosemida, en pacientes con IC avanzada, FEVI deprimida y creatinina $<2,5 \mathrm{mg} / \mathrm{dL}$, demostrando que la asociación consigue mayor 
respuesta clínica (reducción de la disnea y signos congestivos) y un volumen mayor de diuresis que con la administración de altas dosis de furosemida. Además se demostró una disminución de mortalidad por todas las causas y reingresos hospitalarios por insuficiencia cardiaca agudizada, junto con una pérdida significativa de peso preservando la función renal $\left.\right|^{8,9}$

En el Manual práctico de manejo integral del paciente con insuficiencia cardiaca crónica del grupo de la SEMI, se presenta un tabla 1 resumen de las formas de administración del suero salino hipertónico según los niveles de $\mathrm{Na}^{2+}$ en sangre.

Tabla 1: Infusión de SSH en función del valor de $\mathrm{Na}^{2+}$ en sangre

\begin{tabular}{|c|c|c|}
\hline $\begin{array}{l}\text { Concentración } \\
\text { infusión }\end{array}$ & $\begin{array}{l}\text { Valores de } \mathrm{Na}^{2+} \text { en } \\
\text { sangre }\end{array}$ & Forma de preparación \\
\hline $\mathrm{SSH}$ al $4,6 \%$ & $<125 \mathrm{mEq} / \mathrm{L}$ & $\begin{array}{l}\text { Diluir } 19 \text { ampollas de } \mathrm{CINa} \text { al } 20 \% \text { en } 1.000 \mathrm{ml} \text { de } \mathrm{CINa} 0,9 \% \text {; } \\
\text { administrar al paciente } 150 \mathrm{ml} \text { en } 30 \mathrm{~min} / 12 \mathrm{~h}\end{array}$ \\
\hline $\mathrm{SSH}$ al $3,5 \%$ & $126-135 \mathrm{mEq} / \mathrm{L}$ & $\begin{array}{l}\text { Diluir } 14 \text { ampollas de } \mathrm{CINa} \text { al } 20 \% \text { en } 1.000 \mathrm{ml} \text { de } \mathrm{CINa} 0,9 \% \text {; } \\
\text { administrar al paciente } 150 \mathrm{ml} \text { en } 30 \mathrm{~min} / 12 \mathrm{~h}\end{array}$ \\
\hline $\mathrm{SSH}$ al $2,4 \%$ & $>135 \mathrm{mEq} / \mathrm{L}$ & $\begin{array}{l}\text { Diluir } 3 \text { ampollas de } \mathrm{CINa} \text { al } 20 \% \text { en } 1.000 \mathrm{ml} \text { de } \mathrm{CINa} 0,9 \% \text {; } \\
\text { administrar al paciente } 150 \mathrm{ml} \text { en } 30 \mathrm{~min} / 12 \mathrm{~h}\end{array}$ \\
\hline $\mathrm{SSH}$ al $1,4 \%$ & $>145 \mathrm{mEq} / \mathrm{L}$ & $\begin{array}{l}\text { Diluir } 3 \text { ampollas de CINa al } 20 \% \text { en } 1.000 \mathrm{ml} \text { de } \mathrm{CINa} 0,9 \% \text {; } \\
\text { administrar al paciente } 150 \mathrm{ml} \text { en } 30 \mathrm{~min} / 12 \mathrm{~h}\end{array}$ \\
\hline
\end{tabular}

\section{DESARROLLO DE LA EXPERIENCIA}

Presentamos el caso de un paciente de 86 años de edad, institucionalizado con antecedentes de enfermedad pulmonar crónica, cardiopatía isquémica, insuficiencia cardiaca congestiva, enfermedad renal crónica estadio IV y anemia por trastornos crónicos. Es remitido a la Unidad de Hospitalización a Domicilio de San Juan de Alicante por presentar edemas bilaterales hasta tercio medio de ambos muslos, ortopnea y crepitantes en ambas bases a pesar de la administración de dosis elevadas de furosemida (160mg/día).

En nuestra unidad iniciamos perfusión de furosemida (120mg/día) y añadimos clortalidona 25mg/24horas. Los síntomas no desaparecen, por lo que aumentamos clortalidona 50mg/día y la perfusión de furosemida a 180mg/día, consiguiendo una desaparición de los crepitantes y mejoría de la ortopnea. Sin embargo, los edemas seguían produciendo un gran malestar a nuestro paciente.

Ante la persistencia de los edemas a pesar de dosis elevadas de diuréticos, y puesto que el paciente se encuentra institucionalizado en una residencia sociosanitaria, se decide iniciar una perfusión de suero salino hipertónico con 2 ampollas de $\mathrm{NaCl}$ al 20\% (60mEq) en $150 \mathrm{ml}$ de suero salino fisiológico al 0,9\% + $250 \mathrm{mg}$ de furosemida a pasar en 30 minutos a diario, junto a un restricción de agua de $1 \mathrm{~L} /$ día. A los 5 días, al no existir alteración de la función renal ni electrolítica, optamos por suministrarlo cada 12 horas, contando con la ayuda del personal de la residencia.

Realizamos seguimiento clínico y analítico, presentando los resultados en la tabla 2. 
Tabla 2: Registro de datos clínicos y analíticos durante el tratamiento con suero salino hipertónico + furosemida

\begin{tabular}{|c|c|c|c|}
\hline & Pre-tratamiento & $1^{\circ}$ día de tratamiento & $\begin{array}{l}7^{\circ} \text { día de } \\
\text { tratamiento }\end{array}$ \\
\hline Peso (Kg) & 84,3 & 82,1 & 76,1 \\
\hline Pie derecho $(\mathrm{cm})$ & 28 & 27 & 22 \\
\hline Pie izquierdo $(\mathrm{cm})$ & 28 & 27 & 23 \\
\hline Tobillo derecho $(\mathrm{cm})$ & 27 & 26 & 23 \\
\hline Tobillo izquierdo (cm) & 26 & 25 & 22 \\
\hline Pantorrilla derecha $(\mathrm{cm})$ & 35 & 35 & 31 \\
\hline Pantorrilla izquierda $(\mathrm{cm})$ & 36 & 34 & 32 \\
\hline Muslo derecho $(\mathrm{cm})$ & 49 & 48 & 42 \\
\hline Muslo izquierdo (cm) & 48 & 47 & 41 \\
\hline Sensación 1-10 & 10 & 7 & 3 \\
\hline Diuresis (ml/24h) & 1800 & 2400 & 3200 \\
\hline $\mathrm{TA}(\mathrm{mmHg})$ & $130 / 60$ & $110 / 70$ & $115 / 70$ \\
\hline $\mathrm{Na}^{2+}(\mathrm{mmol} / \mathrm{L})$ & 141 & 141 & 143 \\
\hline $\mathrm{K}^{+}(\mathrm{mmol} / \mathrm{L})$ & 3 & 3,10 & 3,10 \\
\hline Creatinina (mg/dL) & 2,68 & 2,55 & 2,27 \\
\hline $\mathrm{FG}(\mathrm{mL} / \mathrm{min})$ & 22,67 & 24 & 27,45 \\
\hline
\end{tabular}

\section{CONCLUSIONES}

En los últimos 30 años, los avances en los tratamientos y su implementación han mejorado la supervivencia y han reducido la tasa de hospitalización en pacientes con Insuficiencia Cardiaca y fracción de eyección reducida, aunque los resultados siguen siendo insatisfactorios ${ }^{10}$

Al administrar suero salino hipertónico, se expande volumen (sobre todo a nivel glomerular), se produce un desplazamiento del agua libre intersticial al compartimento intravascular por lo que la 
hipoperfusión queda contrarrestada y se potencia el efecto del diurético de asa al aportar mucho soluto, consiguiendo un incremento de la diuresis, sin empeoramiento de la función renal. Los resultados de este caso clínico, demuestran que la utilización de furosemida a altas dosis junto con suero salino hipertónico, produce una reducción importante de los edemas en miembros inferiores, entre $15-20 \%$, asociado además a una discreta mejoría de la función renal.

Podemos concluir que en este caso, el tratamiento fue bien tolerado y mejoró la calidad de vida del paciente a través de una mejoría en lo síntomas congestivos.

\section{BIBLIOGRAFÍA}

1. Anguita Sanchez M, Crespo Leiro MG, de Teresa Galvan E, Jimenez Navarro M, Alonso-Pulpon L, Muniz Garcia J, et al. Prevalence of heart failure in the Spanish general population aged over 45 years. The PRICE Study. Rev Esp Cardiol. 2008;61(10):1041-9. PMID: 18817680

2. Roger VL, Go AS, Lloyd-Jones DM, Benjamin EJ, Berry JD, Borden WB, et al. Heart disease and stroke statistics-- 2012 update: a report from the American Heart Association. Circulation. 2012;125(1):e2-e220. DOI: 10.1161/CIR.0b013e31823ac046. PMID: 22179539

3. Rodriguez-Artalejo F, Banegas Banegas JR, Guallar-Castillon P. [Epidemiology of heart failure]. Rev Esp Cardiol. 2004;57(2):163-70. PMID: 14967113

4. Writing Group M, Lloyd-Jones D, Adams RJ, Brown TM, Carnethon M, Dai S, et al. Heart disease and stroke statistics--2010 update: a report from the American Heart Association. Circulation. 2010;121(7):e46-e215. PMID: 20019324 DOI: 10.1161/CIRCULATIONAHA.109.192667

5. Pocock SJ, Ariti CA, McMurray JJV, Maggioni A, Køber L, Squire IB, Swedberg K, Dobson J, Poppe KK, Whalley GA, Doughty RN. Predicting survival in heart failure: a risk score based on 39372 patients from 30 studies. Eur Heart J. 2013;34: 1404-13. PMID: 23095984 DOI: 10.1093/ eurheartj/ehs337. PMID: 23095984 DOI: 10.1093/eurheartj/ehs337

6. Hasselblad V, Gattis Stough W, Shah MR, Lokhnygina Y, O'Connor CM, Califf RM, et al. Relation between dose of loop diuretics and outcomes in heart failure population: results of the ESCAPE trial. Eur J Heart Fail. 2007;9:1064-9. . PMID: 17719273 DOI: 10.1016/j.ejheart.2007.07.011

7. Adams KF Jr, Fonarow GC, Emerman CL, LeJemtel TH, Costanzo MR, Abraham WT, et al. Characteristics and outcomes of patients hospitalized for heart failure in the United States: rationale, design, and preliminary observations from the first 100,000 cases in the Acute Decompensated Heart Failure National Registry(ADHERE). Am Heart J. 2005;149:209-16. PMID: 15846257 DOI: 10.1016/j.ahj.2004.08.005

8. Licata G, Di Pasquale P, Parrinello G, Cardinale A, Scandurra A, Follone G, et al. Effects of highdose furosemide and small-volume hypertonic saline solution infusion in comparison with a high dose of furosemide as bolus in refractory congestive heart failure: long-term effects. Am Heart J. marzo de 2003;145(3):459-66. PMID: 12660669 DOI: 10.1067/mhj.2003.166

9. Gandhi S, Mosleh W, Myers RBH. Hypertonic saline with furosemide for the treatment of acute congestive heart failure: a systematic review and meta-analysis. Int $\mathrm{J}$ Cardiol. 1 de mayo de 2014;173(2):139-45. PMID: 24679680 DOI: 10.1016/j.ijcard.2014.03.020

10. Piotr Ponikowski* (Presidente) (Polonia), Adriaan A. Voors (Copresidente) (Países Bajos), Stefan D. Anker (Alemania), Héctor Bueno (España), et al. Guía ESC 2016 sobre el diagnóstico y tratamiento de la insuficiencia cardiaca aguda y crónica. Rev Esp Cardiol. 2016;69(12):1167.e1-e85 DOI: 10.1016/j.recesp.2016.10.014. 